\title{
Psychological considerations related to the development of computerized testing stations
}

\author{
JAMES H. JOHNSON and KATHY N. JOHNSON \\ Illinois Institute of Technology, Chicago, Illinois 60616
}

\begin{abstract}
A review of pertinent recent events in psychological assessment suggests greater use of computerized testing systems in the future. Just as there are problems of response bias with paper-and-pencil test methods, there are also such problems when using the computer medium for assessment. Problem areas and possible solutions are reviewed. Future research needs are discussed.
\end{abstract}

In 1975, Johnson and Williams reported on the development of a large-scale on-line computer-based testing system for patients at the VA Hospital in Salt Lake City. This was the first major effort to make use of on-line computer terminals for routine psychological testing in a mental health setting. Evaluation results with this system showed that it was cost-effective and, still, well liked by test takers (Klingler, Johnson, \& Williams, 1976; Klingler, Miller, Johnson, \& Williams, 1977). These results were found despite the fact that efforts to human engineer the terminal system were only very rudimentary (Cole, Johnson, \& Williams, 1975).

Concurrent with the development of this operational psychological testing system were many other developments important to on-line computer-based assessment. Among the most important of these was the introduction of adaptive testing methodologies (Dewitt \& Weiss, 1976). Adaptive testing strategies are characterized by large item pools that are only partially administered to each particular test taker, depending on initial item responses and the individual's level of functioning. Because the adaptive approach concentrates on examining primarily in the area of ability appropriate to the individual being tested, it offers the possibility of narrow-band assessment with only a small number of items being presented to any one test taker.

The positive empirical findings by Johnson and his colleagues in Utah and the obvious theoretical advantages of adaptive strategies over other assessment techniques leads us to expect that an ever-growing percentage of future testing packages will be developed for the on-line medium. As a result, it appears that we will be entering a new age of research in psychological assessment. In this era, several new topics that were previously only tangentially considered will emerge as relevant concerns for test constructors. The present paper is concerned with one of these new assessment research areas, human engineering the computerized testing station. Our

Requests for reprints should be sent to James H. Johnson, Department of Psychology, Illinois Institute of Technology, Chicago, Illinois 60616 . purposes in presenting this paper are to review the nature of the research problem, to suggest some approaches for minimizing the problem, and to make some proposals for future research in the area. In essence, we are proposing that efforts need to be taken to bring about "man-computer symbiosis" (Licklider, 1960) in the adaptive testing area and that these efforts will have great importance to the success of this new testing approach.

\section{HUMAN ENGINEERING TESTING STATIONS AS A DESCENDANT OF RESPONSE BIAS}

Some years ago, Edwards (1957) and Jackson and Messick (1961) argued that most of the variance in objective personality testing could be accounted for by response sets. Even though Block (1965) showed this not to be the case, relevant concerns about minimizing the amount of invalid test variance due to the response process remain in the testing area. Figure 1 shows a schematic of the computerized test response process. It is apparent from this schematic that variance due solely to styles of response is only a small part of the invalid variance possible in a testing situation. Errors due to reading and understanding on the input side and due to slips between the thinking process and the physical response movement on the output side are also likely to lead to undesirable test variance.

\section{SCHEMATIC OF THE COMPUTERIZED TESTING PROCESS}

Because of the possibility of errors involving the person-test system interface, it is important to consider ways to human engineer computerized testing stations in order to reduce unwanted variance. One can see that while this problem is a variation on that first considered by Edwards (1957) and then by Jackson and Messick (1961), it is potentially of much greater significance to developers of assessment approaches for the on-line computer medium. Furthermore, it is especially important in adaptive testing due to the fact that each indi- 


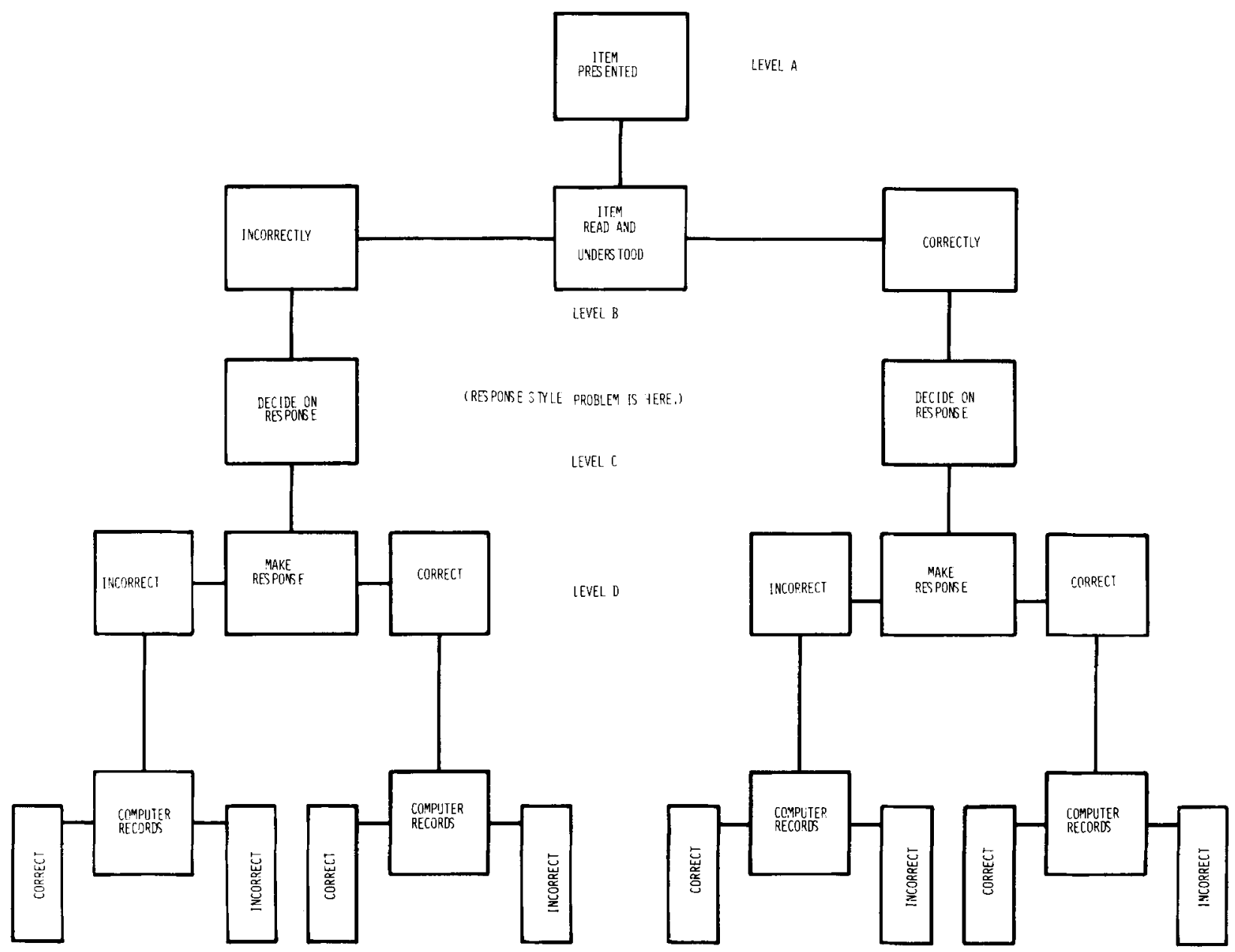

LEVEL E

Figure 1. Schematic of the computerized testing process.

vidual response is directly related to all following item presentations.

\section{EXAMPLES OF HOW TO HUMAN ENGINEER A TESTING STATION}

From Figure 1, it is clear that human engineering a testing station must be completed at both the input and the output levels. Therefore, these problem areas will be treated separately.

\section{Input Problems}

The first possibility of error results from misunderstanding instructions related to taking the test. Greist and Klein (1980) have dealt with this problem by giving very explicit instructions and then testing for understanding. Johnson and Williams (1980) found that, in actual practice, this approach slowed test takers and often led to irritation on their part. They preferred simple instructions with "on-call" personnel to give help if needed. Probably, some compromise is required here. Moderately detailed instructions with simple probing for misunderstanding coupled with "on-call" staff help may be the best solution to assure complete understanding. This problem of whether or not to have highly detailed computerized instructions for the naive user has been considered in detail by a number of researchers (e.g., Kennedy, 1975; Schneiderman, 1980). The concensus of previous research results seems to be that instructions should go from more detailed to less detailed as the user becomes more familiar with usage. Rouse (1977) suggests that the best solution is a dynamic system in which the user chooses the amount of instruction he/she requires. Furthermore, he suggests that a simple game or "trial run" is probably the best way to develop such a dynamic system.

The problems involved in assuring correct reading and understanding of items are more difficult. First, test developers need to refer to the human factors literature to assure that illumination, character size, and item length are optimal for error reduction (e.g., Schneiderman, 1980). Second, they should probably check proposed items with alternates for readability and comprehension before inclusion in a test. Third, they should use some 
kind of screening index, such as the Q1 (Johnson, Williams, Klingler, \& Giannetti, 1977), to predetermine whether a test taker is capable of understanding the item pool. Finally, they may wish to begin work on a new methodology of item construction, one specifically aimed at the on-line medium.

Careful attention to these aspects of computerbased testing can help to reduce many of the errors possible at Level $B$ of the testing process shown in Figure 1.

\section{Output Problems}

Perhaps it is relevant to begin this section with the proverb attributed to Palladus, "There is many a slip twixt the cup and the lip." As is illustrated at Level D of Figure 1, it is possible for the respondent to read an item correctly, make a correct decision about how to respond to the item, and still give an incorrect response. For example, classic human factors research on keyboard usage has shown that even experienced keyboard users have a finger-related error rate ranging from .00414 to .01171 (Hirsch, 1976).

Paying attention to some of this research can help to overcome these problems. From Hirsch's (1976) data, we know that the chances of making an error on Choices "C," "D," and "E" are approximately $50 \%$ greater than they are on Choice "A." Therefore, it seems obvious that there is a need to use specifically designed key pads for response collection. These key pads should be based on the human factors literature and should be designed to minimize error rates, as well as error rate variance across choices.

Moreover, system designers need to take account of the fact that errors will always be made, and, therefore, they should develop simple error correction procedures. A "backup key" on the keyboard is one good solution. If a respondent recognized that he/she may have made an error, then pushing the "backup" key will allow typing the item again.

There should also be a procedure whereby errors in recording by the computer can be made apparent to the test taker for final correction. These errors, at Level $E$ of Figure 1, are not frequent, but they are still certain to occur as a result of hardware and software failure.

What can be done about these errors? One acceptable approach is for the computer to randomly review answers with the respondent at the completion of testing. If the respondent does not agree with this review, then this fact could be reported to invalidate results.

\section{Problems Related to Both Input and Output}

The most obvious problems related to both input and output are those of attitude and anxiety (Schneiderman, 1980). Subjects with negative attitudes toward computers have been shown to perform more slowly and to make more errors (Walther \& O'Neil, 1974). Anxiety also has been related to poorer performance (Denny, 1966). Thus, adaptive test constructors need to work carefully to reduce poor attitudes and high anxiety.

Schneiderman (1980) has further noted that the issues of control and response time are related to attitude and anxiety. He cites evidence that users feel worse about interactive computer systems when they lack control over them. Therefore, careful attention should be given to developing an interactive dialogue that allows the user to feel as though he/she is in charge of the situation. Wide variance in the amount of time taken by the computer to respond to the user, as well as extended response latencies, has also been related to poorer attitudes, high anxiety, and consequent poorer performance (Miller, 1977). Thus, every effort should be made by adaptive systems developers to use approaches that lead to fast and consistent response latencies. This is an especially difficult problem to overcome because adaptive testing requires calculations between each item presentation, and the calculations become more difficult after more items have been presented, thus taking more time. One solution to this problem is to use a fixed latency that is as long as the most difficult item selection computation will take. Of course, this solution is at odds with the need to have generally brief latencies, but it may be the best possible answer to the problem.

\section{FUTURE RESEARCH}

It is now beginning to be clear that the problems associated with the person-computerized testing station interface are numerous. Furthermore, it is evident that psychologists working in the psychometric area have not paid enough attention to these problems. A greater understanding of human factors issues is going to be required in this area.

To begin with, it will be important to know the normal error rate at each of the levels in Figure 1. This knowledge will allow us to test newly developed strategies for improvement in error rate. The development and testing of various new strategies for error reduction probably constitutes the largest research need in this area. For example, one such problem deserves immediate attention. Is a one-key or two-key (the second a RETURN to allow reviewing results) approach better for data entry and error detection? As another example, it might be that different personality states are related to positive effects from different instruction sets. Use of a computerized testing station could begin with a brief screening test that would determine whether one or another set of instructions would have better results. By following the diagrams presented in Figure 1, it can be seen that other research problems in this area are numerous and deserve the attention of automated test developers. 


\section{REFERENCES}

BLock, J. The challenge of response sets. New York: AppletonCentury-Crofts, 1965.

Cole, E. B., Johnson, J. H., \& Williams, T. A. Design considerations for an on-line computer system for automated psychiatric assessment. Behavior Research Methods \& Instrumentation, 1975, 7, 199-200.

Denny, J. P. Effects of anxiety and intelligence on concept formation. Journal of Experimental Psychology, 1966, 72, 596.

DEwiTT, L. J., \& WEISs, D. J. Hardware and software evolution of an adaptive ability measurement system. Behavior Research Methods \& Instrumentation, 1976, 8, 104-107.

Edwards, A. The social desirability in personality assessment research. New York: Holt, 1957.

GREIST, J. H., \& KLEIN, M. H. Computer programs for patients, clinicians, and researchers in psychiatry. In J. B. Sidowski, J. H. Johnson, \& T. A. Williams (Eds.), Technology in mental health care delivery systems. Norwood, N.J: Ablex, 1980.

Hinsch, R. S. Human factors in man computer interfaces. San Jose, Calif: IBM Human Factors Center, 1976.

Jackson, D., \& Messick, S. Acquiescence and desirability determinants in the MMPI. Educational and Psychological Measurement, 1961, 22, 771-790.

Johnson, J. H., \& Williams, T. A. The use of on-line computer technology in a mental health admitting system. American Psychologist, 1975, 30, 388-390.

Johnson, J. H., \& Williams, T. A. Using on-line computer technology to improve service response and decision-making effectiveness in a mental health admitting system. In J. B. Sidowski, J. H. Johnson, \& T. A. Williams (Eds.), Technology in mental health care delivery systems. Norwood, N.J: Ablex, 1980.

Johnson, J. H., \& Williams, T. A., Klingler, D. E., \& Giannetri, R. A. Interventional relevance and retrofit programming: Concepts for the improvement of clinician acceptance of computer-generated assessment reports. Behavior Research Methods \& Instrumentation, 1977, 9, 123-132.

KENNEDY, T. C. Some behavioral factors affecting the training of naive users of an interactive computer system. International Journal of Man-Machine Studies, 1975, 1, 817-834.

Klingler, D. E., Johnson, J. H., \& Williams, T. A. Strategies in the evaluation of an on-line computer-assisted unit for intake assessment of mental health patients. Behavior Research Methods \& Instrumentation, 1976, 8, 95-100.

Klingler, D. E., Miller, D. A., Johnson, J. H., \& Williams, T. A. Process evaluation of an on-line computer-assisted unit for intake assessment of mental health patients. Behavior Research Methods \& Instrumentation, 1977, 9, 110-116.

Licklider, J. C. Man-computer symbiosis. IEEE Transactions on Human Factors in Electronics, 1960, HFEI, 4-11.

Miller, L. H. A study in man-machine interaction. Proceedings of the National Computer Conference, 1977, 46, 409-421.

Rouse, W. B. Human-computer interaction in multitask situations. IEEE Transactions on Systems, Man and Cybernetics, 1977, 5, 384-392.

Schneiderman, B. Software psychology. Cambridge, Mass: Winthrop, 1980.

Walther, G. H., \& O'Neıl, H. F. On-line user-computer interface: The effects of interface flexibility, terminal type, and experience on performance. Proceedings of the National Computer Conference, 1974, 43, 379-384. 